\title{
Virtual satellite observations of plasmoids generated by fast reconnection in the geomagnetic tail
}

\author{
M. Ugai \\ Research Center for Space and Cosmic Evolution, Ehime University, Matsuyama 790-8577, Japan \\ Received: 20 April 2011 - Revised: 14 June 2011 - Accepted: 1 August 2011 - Published: 23 August 2011
}

\begin{abstract}
The present paper studies fundamental features of plasmoid propagation by virtual satellite observations in the simulation box. The plasmoid domain is divided into the plasmoid reconnection region $\mathrm{P}$, where magnetized plasmas with reconnected field lines, heated by dissipation mechanisms of fast reconnection, are accumulated, and the plasmoid core region $\mathrm{C}$, where magnetized plasmas with sheared field lines, initially embedded in the current sheet, is adiabatically compressed. When the virtual satellite is located in a position through which the plasmoid core region passes, it detects distinct changes in quantities at the interface between the regions $\mathrm{P}$ and $\mathrm{C}$, where the north-south field component $B_{\mathrm{Z}}$ has the bipolar profile and the sheared field component $B_{\mathrm{y}}$ has the peak value. The observed magnetic field profile is, both quantitatively and qualitatively, in good agreement with the standard one detected by actual satellite observations, although when the satellite location is very close to the $X$ neutral line, where reconnection occurs, the $B_{\mathrm{Z}}$ field profile becomes dipolarization-like rather than bipolar. If the satellite detects only the plasmoid region $\mathrm{P}$ outside region $\mathrm{C}$, the standard magnetic field profile becomes obscure even if notable plasmoid signatures, such as enhanced plasma temperature and plasma flow, are observed. Unlike the traditional flux rope model based on multiple reconnections, it is demonstrated that the standard magnetic field profile, observed for plasmoids propagating in the geomagnetic tail, is the direct outcome of the single fast reconnection evolution.
\end{abstract}

Keywords. Magnetospheric physics (Magnetotail; Storms and substorms)

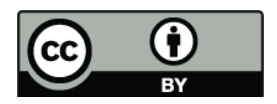

Correspondence to: M. Ugai

(ugai@ cosmos.ehime-u.ac.jp)

\section{Introduction}

In space plasmas, magnetic reconnection is essential for large dissipative events, such as solar flares and geomagnetic substorms (Priest and Forbes, 2000; Runov et al., 2003; Nakamura et al., 2006), and the so-called fast reconnection mechanism, involving slow shocks and Alfvénic plasma jets, should be most applicable to substorms (Petschek, 1964). Hence, the key question is to clarify how the fast reconnection mechanism can be realized as an eventual solution. Historically, possible fast reconnection configurations were proposed for two-dimensional (2-D) steady magnetohydrodynamic (MHD) flows, and it was argued that the fast reconnection mechanism should be controlled by external boundary conditions (Petschek, 1964; Vasyliunas, 1975; Priest and Forbes, 1986). On the other hand, Ugai and Tsuda (1977) first demonstrated that the fast reconnection mechanism can spontaneously be realized if an anomalous resistivity is localized in the current sheet. They demonstrated that the $\boldsymbol{J} \times \boldsymbol{B}$ force resulting from magnetic reconnection drives the plasma flow so as to vitally concentrate the current density into the diffusion region to enhance the reconnection evolution. This remarkable reconnection dynamic results from the basic properties of current sheet systems in collisionless (space) plasmas.

On the basis of their results, we have proposed the spontaneous fast reconnection model. The details of this model are described in Ugai $(2008,1999,1992)$ and references therein. In this model, the fast reconnection mechanism evolves by a nonlinear instability due to positive feedback between current-driven anomalous resistivities and global reconnection flows. In three-dimensional (3-D) current sheet systems without initial sheared field, the key physical condition for the fast reconnection evolution is that the current sheet width in the sheet's current direction is about three times larger than its thickness. Once such a thin current sheet is formed in collisionless plasmas, initiated by a reconnection

Published by Copernicus Publications on behalf of the European Geosciences Union. 


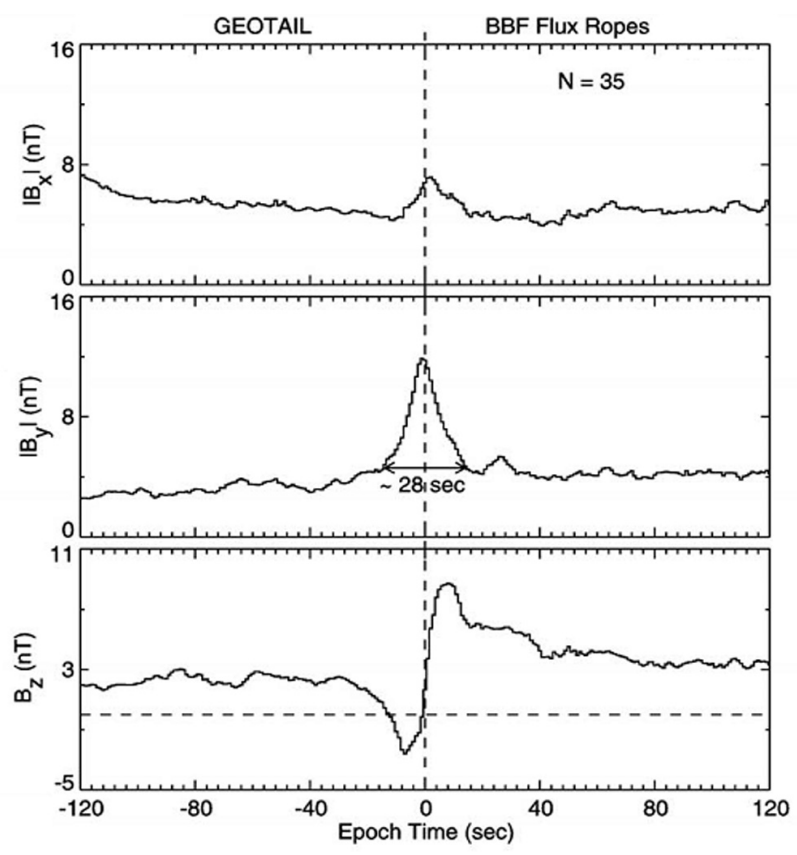

Fig. 1. Superposed epoch analysis performed using $1 \mathrm{~s}$ averages of the Geotail magnetic field measurements for all $35 \mathrm{BBF}$ flux rope events (after Slavin et al., 2003).

disturbance, extreme current sheet thinning (current concentration) occurs around an $X$ neutral line, which cannot be suppressed by MHD effects (Ugai, 1986, 2008). According to the extreme current concentration, some dissipation mechanism (effective resistivity) in the form of $\boldsymbol{E} \cdot \boldsymbol{J}>0$ should be caused around the $X$ line. In generalized Ohm's law, the Hall term does not provide any dissipation and electron inertial effects are considered to cause a dissipation. However, the inertial effects work so as to short-circuit the reconnection electric field $\boldsymbol{E}$, so the current sheet thinning could not effectively be suppressed. Hence, it is reasonable to consider that the current sheet thinning drastically proceeds, until currentdriven anomalous resistivities are necessarily caused in collisionless plasmas (Lui, 2001, 2004; Petkaki and Freeman, 2008). In fact, in laboratory experiments, such current-driven anomalous resistivities are definitely detected around the $X$ neutral line (Ono et al., 2001; Ji et al., 1998). We then find that the fast reconnection evolution is neither influenced by the functional form nor the parameter value of current-driven anomalous resistivity model. In other words, the fast reconnection mechanism can readily build up spontaneously in such thin current sheets once they are formed in space plasmas.

The reconnection dynamics involves complicated nonlinear plasma processes, so computer simulations have played an important role. However, in performing reconnection simulations, special care must be paid to the physical dissipation (effective resistivity) in the $X$ reconnection (diffusion) region. In fact, since in collisionless plasmas extreme current concentration occurs in the diffusion region, if physical dissipations are not sufficiently large, magnetic reconnection drastically occurs by numerical (artificial) dissipations. For MHD simulations, if the resistivity formulation is specified explicitly, the numerical resistivity involved can directly be checked (Ugai, 2008). For particle simulations, the effective resistivity is frequently provided by electron inertial effects, but the inertial resistivity must be zero in the limit of zero electron mass, $m_{\mathrm{e}}=0$. Hence, it is essential to check how the inertial resistivity becomes reduced by reducing $m_{\mathrm{e}}$, which requires a sufficiently large number of particles to always be retained in the reduced electron diffusion region of electron inertial length. It should be noted that numerical resistivities usually cause drastic buildup of magnetic reconnection, leading to, for instance, magnetic islands, which might look fit for some substorm observations, but they cannot be applied to actual phenomena.

Any theoretical model responsible for substorms must reasonably explain fundamental observational features. Geomagnetic substorms, where magnetic reconnection may occur in a simple current sheet configuration, can directly be measured by in situ satellite observations. In particular, socalled plasmoids are known to propagate in the geomagnetic tail in accordance with substorm onset, and it is the longstanding question to clarify the physical mechanism. If a magnetic neutral sheet is formed somewhere in the near-earth tail plasma sheet, magnetic reconnection may suddenly be triggered to give rise to a pair of plasmoids propagating in the tailward and earthward directions, since magnetic reconnection itself occurs symmetrically. In the classical model, the tailward moving plasmoid is considered to be formed between the reconnected field lines and the pre-existing northward field lines (Hones Jr., 1977), so that the field lines inside the plasmoid will be closed (or helical). Regarding the earthward moving plasmoid, Fig. 1 shows the typical satellite observations, which indicate that the field component $B_{\mathrm{Z}}$ has the bipolar structure when the sheared field component $B_{\mathrm{y}}$ has the peak value (Slavin et al., 2003). This characteristic magnetic field profile may be called the standard field profile in this paper, since it is also observed for tailward moving plasmoids (Ieda et al., 1998). On the basis of this field profile, the plasmoid is supposed to be a (force-free) flux rope with helical field lines generated by multiple reconnections.

On the other hand, we recently studied the plasmoid structure and dynamics, generated by the fast reconnection mechanism, and demonstrated that the field lines inside the plasmoid are not helical nor closed (Ugai, 2010, 2011). A similar conclusion is also suggested on the basis of satellite observations (Sormakov and Sergeev, 2008; Sergeev et al., 1992). Hence, the main theme of the present paper is to examine this controversial issue by virtual satellite observations. On the basis of Ugai (2011), Fig. 2a shows the schematic drawing of a plasmoid travelling in the earthward direction, where the $\mathrm{x}-, \mathrm{y}-$ and $\mathrm{z}$-axes are directed to the earthward, duskward 
and northward directions, consistent with the GSM coordinates; also, the origin $x=0$ is taken to be the initial $X$ line location, where magnetic reconnection starts. Figure $2 \mathrm{~b}$ shows the magnetic field lines passing through the plasmoid, which indicate that they are not helical inside the plasmoid. In this case, the region $\mathrm{P}$ above and below region $\mathrm{C}$ is inversely shifted in the $\mathrm{y}$-direction to bend the field lines accumulated in region $\mathrm{C}$, marked by the letter $B$, in the z-direction (Ugai, 2011).

In this paper, the "plasmoid" means such a plasma sheet bulge that is directly generated by the fast reconnection jet, which was first reported by Ugai $(1989,1995)$ and extensively studied (Nitta, 2004; Zenitani and Miyoshi, 2011); in fact, magnetic islands, usually generated in the active reconnection region, may also propagate as smaller-scale plasmoids in the geomagnetic tail, which are not considered here. Also, in the present model, $B_{\mathrm{Z}}=0$ is assumed in the initial current sheet, so that the northward field component $B_{\mathrm{Z}}>0$, which exists in the tail plasma sheet outside the magnetic neutral sheet, is not considered.

\section{Simulation modelling}

The present simulation model is the same as Ugai (2011), and all the details of the numerical procedure are already described in Ugai (2008), which assures us that throughout the present computations the numerical resistivity involved is much smaller than the physical resistivity. All the present simulation results can readily be confirmed on the basis of Ugai (2008).

\subsection{Basic equations}

The compressible MHD equations are

$D \rho / D t=-\rho \nabla \cdot \boldsymbol{u}, \quad \rho D \boldsymbol{u} / D t=-\nabla P+\boldsymbol{J} \times B$,

$\partial \boldsymbol{B} / \partial t-\nabla \times(\boldsymbol{u} \times B)=-\nabla \times(\eta \boldsymbol{J})$,

$\rho D e / D t=-P \nabla \cdot \boldsymbol{u}+\eta \boldsymbol{J}^{2}$,

$\boldsymbol{J}=\nabla \times \boldsymbol{B} / \mu_{0}, \quad \nabla \cdot \boldsymbol{B}=0$,

where $D / D t \equiv \partial / \partial t+\boldsymbol{u} \cdot \nabla$; the gas law, $P=(\gamma-1) \rho e$, is assumed [ $e$ is the internal energy per unit mass, and $\gamma$ is the specific heat ratio with $\gamma=5 / 3$ assumed here (an adiabatic case)], as is Ohm's law, $\boldsymbol{E}+u \times B=\eta \boldsymbol{J}$ ( $\eta$ may be an effective resistivity). The basic Eqs. (1) are transformed to a conservation-law form and the modified Lax-Wendroff scheme is used for the numerical computation (Ugai, 2008).

\subsection{Initial-boundary conditions}

Initially, a long current sheet with sheared antiparallel magnetic fields $\left(B_{\mathrm{X}}\right)$ is assumed. Then, the normalization of
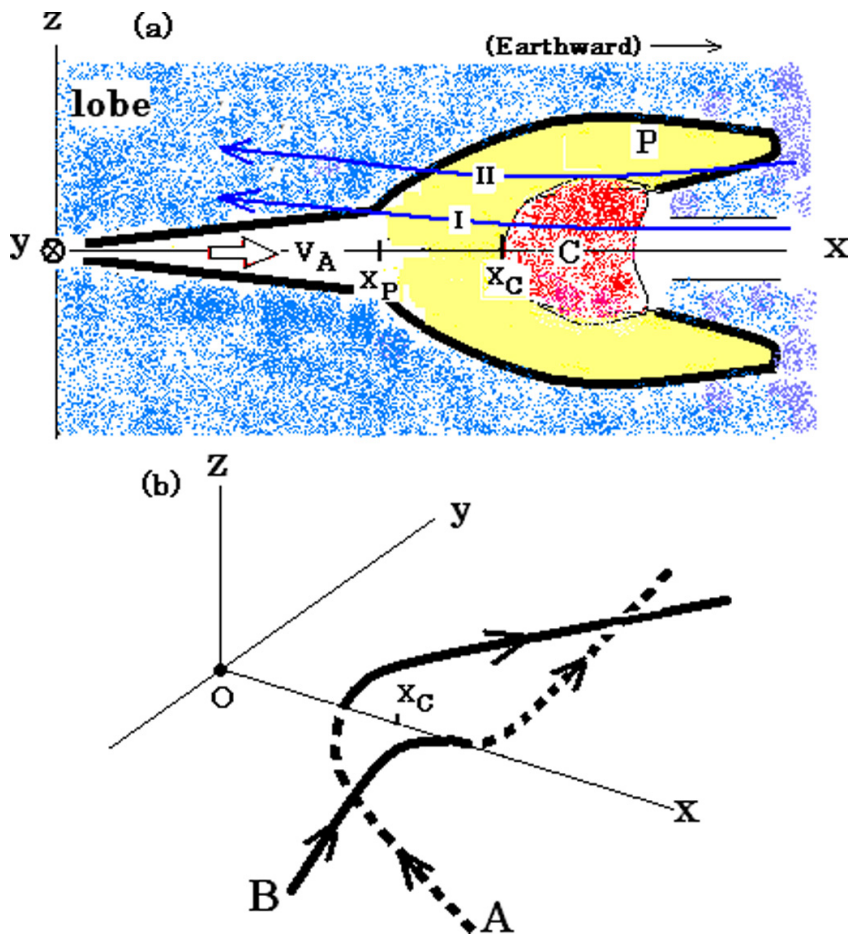

Fig. 2. (a) Schematic drawing of a plasmoid propagating in the positive $\mathrm{x}$-directions in the $y=0[(x, z)]$ plane, where the plasmoid domain is divided into the plasmoid reconnection region $\mathrm{P}$ and the plasmoid core region $\mathrm{C}$, where $X_{\mathrm{P}}$ and $X_{\mathrm{C}}$ indicate, respectively, the plasmoid backward end and the plasmoid centre; (b) the reconnected field lines denoted by $A$ in $x<X_{\mathrm{C}}$ and the accumulated sheared field lines denoted by $B$ in $x>X_{\mathrm{C}}$. In (a) typical satellite observation trajectories are indicated by I and II.

quantities, based on the initial quantities, is self-evident: Distances are normalized by the $\mathrm{z}$-directional current sheet halfthickness $d_{0}$, magnetic field $\boldsymbol{B}$ by $B_{\mathrm{x} 0}$, plasma pressure $P$ by $P_{0}=B_{\mathrm{x} 0}^{2} /\left(2 \mu_{0}\right)$; also, plasma density $\rho$ by $\rho_{i}=\rho(z=0)$, plasma flow velocity $\boldsymbol{u}$ by $V_{\mathrm{Ax} 0}\left(=B_{\mathrm{x} 0} / \sqrt{\mu_{0} \rho_{i}}\right)$, time $t$ by $d_{0} / V_{\mathrm{Ax} 0}$. The Alfvén velocity based on $B_{\mathrm{x} 0}$ in the ambient magnetic field region is given by $V_{\mathrm{Ae}}=V_{\mathrm{Ax} 0} / \sqrt{\rho_{\mathrm{e}}}\left(\rho_{\mathrm{e}}\right.$ is the plasma density in the magnetic field region).

The magnetic field $\boldsymbol{B}=\left[B_{\mathrm{x}}(z), B_{\mathrm{y}}(z), 0\right]$ is initially assumed as: $B_{\mathrm{X}}(z)=\sin (\pi z / 2)$ for $0<z<1 ; B_{\mathrm{X}}=1$ for $1<z<5 ; B_{\mathrm{x}}=\cos [(z-5) \pi / 0.6]$ for $5<z<5.3 ; B_{\mathrm{x}}=0$ for $z>5.3$; also, $B_{\mathrm{X}}(z)=-B_{\mathrm{X}}(-z)$ for $z<0$. The sheared field component $B_{\mathrm{y}}$ is initially assumed as: $B_{\mathrm{y}}(z)=B_{\mathrm{y} 0}+$ $\alpha \cos (z \pi / 2)$ for $|z|<1$ and $B_{\mathrm{y}}=B_{\mathrm{y} 0}$ for $|z|>1$. Here, $B_{\mathrm{y} 0}$ and $\alpha$ are taken to be parameters for the initial sheared field. Fluid velocity $\boldsymbol{u}=(0,0,0)$, and plasma pressure $P(z)$ satisfies the pressure-balance condition,

$P+B_{\mathrm{x}}^{2}+B_{\mathrm{y}}^{2}=1+\beta_{0}+B_{\mathrm{y} 0}^{2}$,

where $\beta_{0}$ is the ratio of the plasma pressure to the magnetic pressure $B_{\mathrm{x} 0}^{2} /\left(2 \mu_{0}\right)$ in the magnetic field region $1<z<5$ 


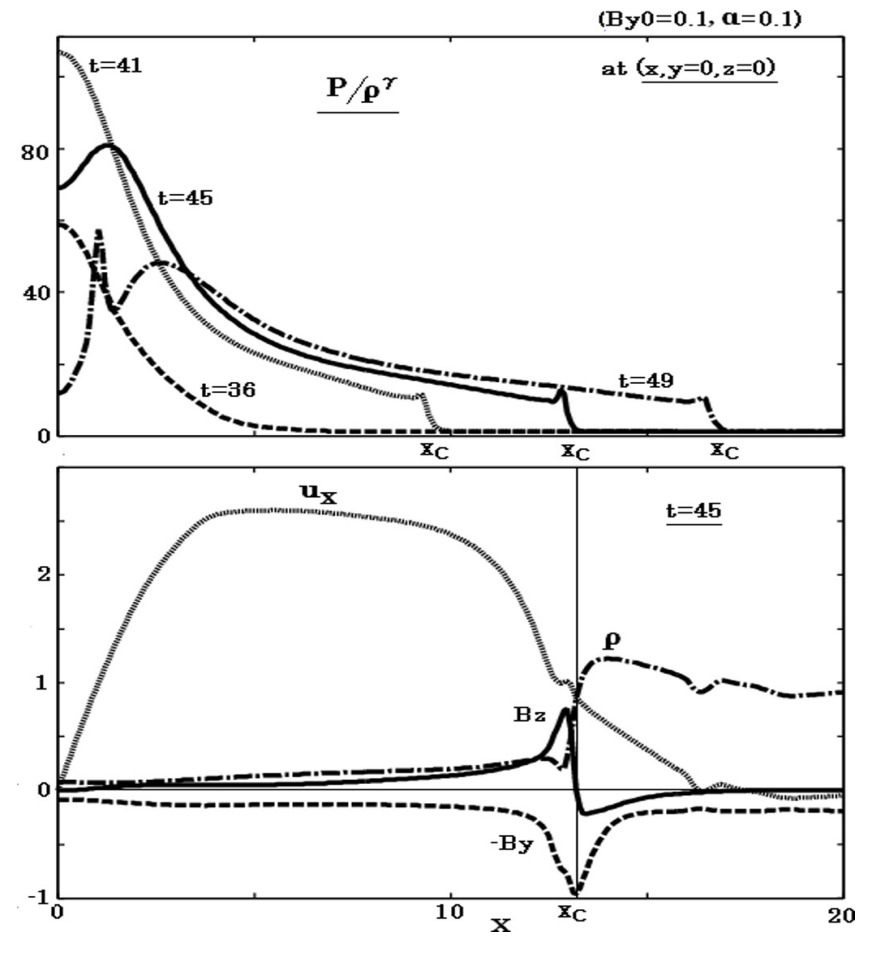

Fig. 3. Profiles of $P / \rho^{\gamma}$ along the $\mathrm{x}$-axis at different times $(\gamma=$ $5 / 3)$ and those of $\rho, u_{\mathrm{X}},-B_{\mathrm{y}}$ and $B_{\mathrm{Z}}$ at $t=45$ along the $\mathrm{x}$-axis, where $X_{\mathrm{C}}$ is the plasmoid centre, for the case of $B_{\mathrm{y} 0}=0.1$ and $\alpha=0.1$.

(here, $\beta_{0}=0.15$ is taken). Also, plasma density $\rho$ initially satisfies

$\rho(z)=P(z) /\left(1+\beta_{0}+B_{\mathrm{y} 0}^{2}-\left(B_{\mathrm{y} 0}+\alpha\right)^{2}\right)$,

so that $\rho_{\mathrm{e}}=\beta_{0} /\left(1+\beta_{0}+B_{\mathrm{y} 0}^{2}-\left(B_{\mathrm{y} 0}+\alpha\right)^{2}\right)$ in the ambient magnetic field region, and the initial temperature $T=P / \rho$ is, for simplicity, assumed to be constant everywhere. The computational region is taken to be a rectangular box, $0 \leq$ $x \leq L_{\mathrm{x}}, 0 \leq z \leq L_{\mathrm{z}}$, and $-L_{\mathrm{y}} \leq y \leq L_{\mathrm{y}}$, and the axis symmetry boundary conditions are assumed with respect to the $\mathrm{x}$ - and z-axes (Ugai, 2011). On the other boundary planes $\left(x=L_{\mathrm{x}}, y=L_{\mathrm{y}}, y=-L_{\mathrm{y}}, z=L_{\mathrm{z}}\right)$, the free boundary conditions are assumed, so that the reconnection ouflow is open to the free space.

The effective resistivity for the Ohm's law (normalized by $\left.\mu_{0} d_{0} V_{\mathrm{Ax} 0}\right)$ is assumed to be,

$$
\begin{aligned}
\eta(\boldsymbol{r}, t) & =k_{\mathrm{d}}\left[V_{\mathrm{d}}(\boldsymbol{r}, t)-V_{\mathrm{C}}\right] \quad \text { for } V_{\mathrm{d}}>V_{\mathrm{C}}, \\
& =0 \quad \text { for } V_{\mathrm{d}}<V_{\mathrm{C}}
\end{aligned}
$$

where $V_{\mathrm{d}}(\boldsymbol{r}, t)=|\boldsymbol{J}(\boldsymbol{r}, t) / \rho(\boldsymbol{r}, t)|$ is the relative electron-ion drift velocity. Here, we take $k_{\mathrm{d}}=0.003$ and $V_{\mathrm{C}}=12$ in Eq. (4) as before. Also, in order to disturb the initial configuration, a localized resistivity is assumed around the origin $(\boldsymbol{r}=0)$ in the form,

$\eta(\boldsymbol{r})=\eta_{0} \exp \left[-\left(x / k_{\mathrm{x}}\right)^{2}-\left(y / k_{\mathrm{y}}\right)^{4}-\left(z / k_{\mathrm{z}}\right)^{4}\right]$, which causes magnetic reconnection as an initial disturbance to break the initial static condition. Here, we take $\eta_{0}=0.02$ and $k_{\mathrm{x}}=k_{\mathrm{z}}=0.8$, and $k_{\mathrm{y}}=5$. This disturbance (5) is imposed only in the initial time range $0<t<4$, and the resistivity model (4) is assumed for $t>4$.

The parameters for the numerical computations are taken to be $L_{\mathrm{x}}=20, L_{\mathrm{z}}=7.2$, and $L_{\mathrm{y}}=10$, and the mesh sizes are $\Delta x=0.04, \Delta z=0.015$ and $\Delta y=0.1$.

\section{Results}

In previous papers (Ugai, 2010, 2011), the structure and dynamics of the plasmoid generated by the fast reconnection mechanism are generally studied for three cases (1) $B_{\mathrm{y} 0}=$ 0.1 and $\alpha=0.1$, (2) $B_{\mathrm{y} 0}=0.1$ and $\alpha=0.2$ and (3) $B_{\mathrm{y} 0}=0.2$ and $\alpha=0$. In the present paper, these simulation results are employed for virtual satellite observations. First of all, some basic features of the plasmoid structure, which are needed for the satellite observations, are shown for the typical case of $B_{\mathrm{y} 0}=0.1$ and $\alpha=0.1$ as before, since the basic plasmoid structure is not influenced by the parameter values. Then, observations by virtual satellites, located along some characteristic trajectories, are demonstrated mainly for the typical case of $B_{\mathrm{y} 0}=0.2$ and $\alpha=0$.

\subsection{Basic plasmoid structure}

As shown in Fig. 2a, the overall plasmoid structure is divided into the plasmoid reconnection region $\mathrm{P}$ and the plasmoid core region $C$ (Ugai, 2011). In region $P$, magnetized plasmas with the reconnected field lines, which were heated by dissipations of the fast reconnection mechanism, are accumulated. In region $\mathrm{C}$, magnetized plasmas with the sheared field lines initially embedded in the central plasma sheet, which were ejected by the reconnection jet and adiabatically compressed, are accumulated without reconnection. In Fig. 2b, the reconnected sheared field lines in $x<X_{\mathrm{C}}$, denoted by $A$, that pass through region $\mathrm{P}$, and the sheared field lines without reconnection in $x>X_{\mathrm{C}}$, denoted by $B$, that pass through region $\mathrm{C}$ are shown. The plasmoid centre $X_{\mathrm{C}}$ corresponds to the so-called contact discontinuity that bounds the regions $\mathrm{P}$ and C. In order to see this more clearly, Fig. 3 shows the profiles of the quantity $P / \rho^{\gamma}$ along the x-axis at different times $(\gamma=5 / 3)$; similarly, profiles of plasma quantities and magnetic fields are shown at time $t=45$. Since $P / \rho^{\gamma}$ does not change by convection if plasma experiences no physical dissipation, the distinct plasmoid compression in $x>X_{\mathrm{C}}$, where $P / \rho^{\gamma}$ remains constant, occurs adiabatically, whereas in $x<X_{\mathrm{C}}$ strong non-adiabatic heating occurs. The nonadiabatic heating is found to be most distinct around the $X$ neutral line, so the peak around $x=2$ at $t=49$ indicates that a new $X$ line is located there because of the secondary tearing.

Note that the region $\mathrm{P}$ is characterised by the hightemperature plasmas that has been heated nonadiabatically, 

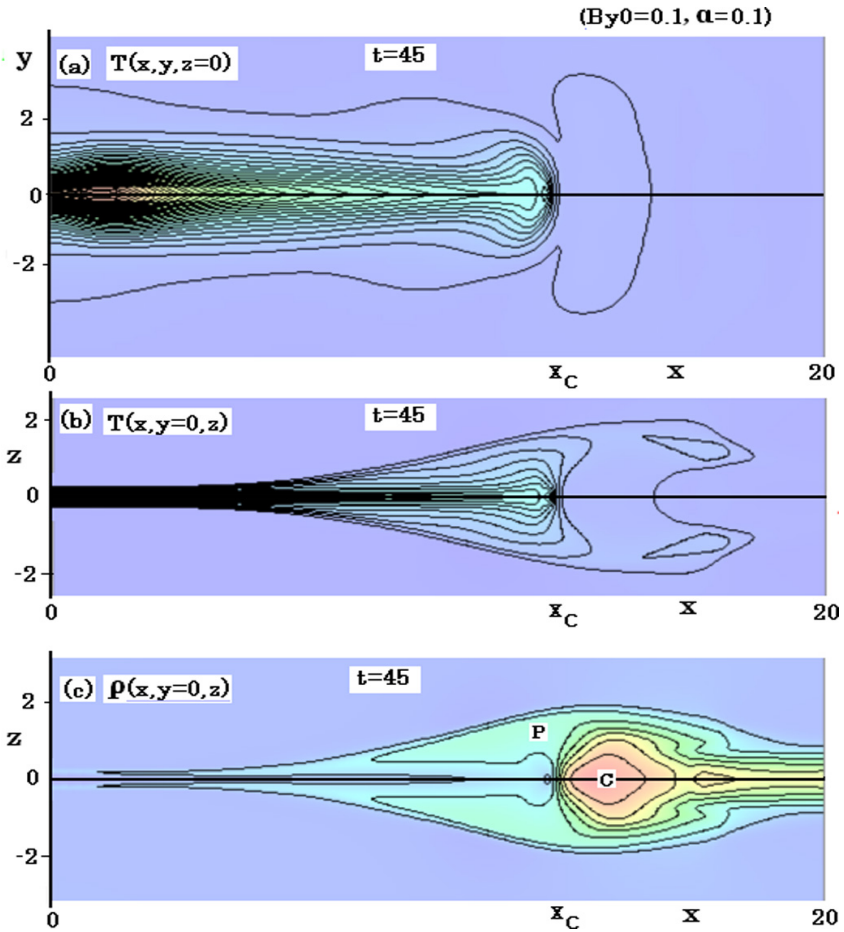

Fig. 4. Plasma temperature distributions with a contour interval of 0.4 (a) in the $z=0$ plane and (b) in the $y=0$ plane, and (c) plasma density distribution with a contour interval of 0.15 in the $y=0$ plane, at time $t=45$ for the case of $B_{\mathrm{y} 0}=0.1$ and $\alpha=0.1$, where $X_{\mathrm{C}}$ is the plasmoid centre and the plasmoid regions $\mathrm{P}$ and $\mathrm{C}$ are indicated.

whereas region $\mathrm{C}$ is characterised by the high-density plasmas due to adiabatic compression. Hence, Fig. 4 shows the distributions of the temperature $T$ in the $y=0$ and $z=0$ planes and those of plasma density $\rho$ in the $y=0$ plane at $t=45$ for the case of $B_{\mathrm{y} 0}=0.1$ and $\alpha=0.1$. We see that the temperature $T$, which was initially constant $(=1.12) \mathrm{ev}$ erywhere, is extremely enhanced in the region where reconnected magnetized plasmas accumulate. Also, the fast reconnection region of high temperature remains to be significantly limited in a narrow channel in the y-direction (Ugai, 2010, 2011), and the plasma density $\rho$ is distinctly enhanced in region C. Figure 5 shows the distributions of the temperature $T$ and the plasma density $\rho$ in the $y=1$ and $y=-1$ planes at $t=45$, which indicates that the plasmoid region $P$ has shifted in the positive (negative) y-direction for $z>0(z<$ $0)$; accordingly, region $\mathrm{C}$ is bent in the $\mathrm{z}$-direction as shown in Fig. 2b (Ugai, 2011).

An actual satellite located in the geomagnetic tail will detect changes in quantities along a trajectory across the plasmoid as it travels in the positive $\mathrm{x}$-direction. In view of the basic plasmoid structure (Fig. 4), the characteristic satellite observation trajectories may typically be divided into the two types, I and II, as shown in Fig. 2a. In the trajectory I, the satellite may be located in the inner part of the plasmoid
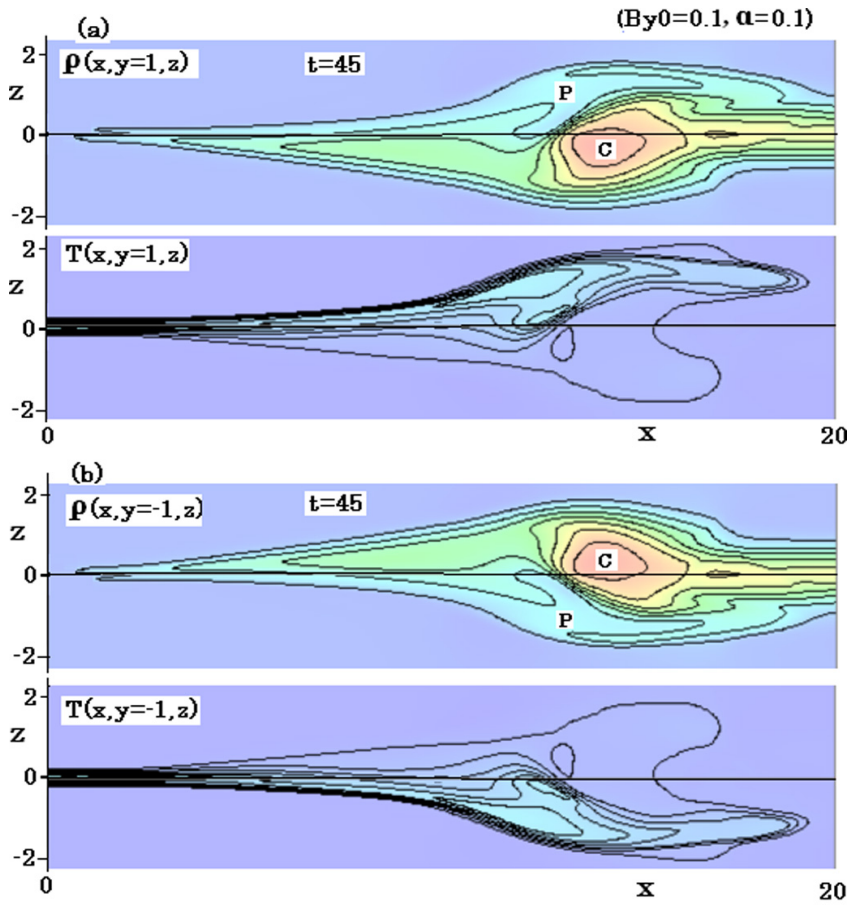

Fig. 5. Plasma density (contour interval of 0.15 ) and temperature (contour interval of 0.4 ) distributions in the (a) $y=1$ plane and (b) $y=-1$ plane, at time $t=45$ for the case of $B_{\mathrm{y} 0}=0.1$ and $\alpha=$ 0.1 , where the plasmoid regions $\mathrm{P}$ and $\mathrm{C}$ are indicated.

(smaller $|z|$ ) and observe region $\mathrm{C}$ and then region $\mathrm{P}$. In the trajectory II, the satellite may be located in the upper part of the plasmoid (larger $|z|$ ) and observe only region $\mathrm{P}$, or the regions $\mathrm{P}, \mathrm{C}$ and $\mathrm{P}$. Regarding the typical structure along the trajectory I, Fig. 6 shows the x-directional profiles of magnetic fields and plasma quantities at $y=0$ and $z=0.45$ (Fig. 4). Inside the plasmoid, the $B_{\mathrm{x}}$ field decreases and the temperature $T$ and fluid velocity $u_{\mathrm{x}}$ increase in region $\mathrm{P}$, whereas in region $\mathrm{C}$ both $T$ and $u_{\mathrm{x}}$ decrease and the plasma density $\rho$ increases. Also, notable changes in quantities occur at the plasmoid boundaries (at $x \sim 6$ and $x \sim 17$ ). Similarly, Fig. 7 shows those of magnetic fields and plasma quantities at $y=0$ and $z=1.35$, which may provide the typical structure along the trajectory II across the regions $\mathrm{P}, \mathrm{C}$ and $\mathrm{P}$.

Distinct changes in quantities occur at the interface between the regions $\mathrm{P}$ and $\mathrm{C}$ inside the plasmoid. Along the $\mathrm{x}$-axis, which may be considered to be the plasmoid central axis, this interface is identified with the plasmoid centre $X_{\mathrm{C}}$. In fact, as seen from Fig. 3 , it is at $x=X_{\mathrm{C}}$ that the field component $B_{\mathrm{Z}}$ changes its sign and the $B_{\mathrm{y}}$ field has the peak value. However, at larger distances from the plasmoid central axis (x-axis), the interface may become more obscure, and the standard magnetic field profile (Fig. 1) cannot be clearly seen even if there are still significant plasma flows. For instance, Fig. 8 shows the x-directional profiles of quantities at $y=2$ and $3(z=0.6)$, which indicate that inside the 


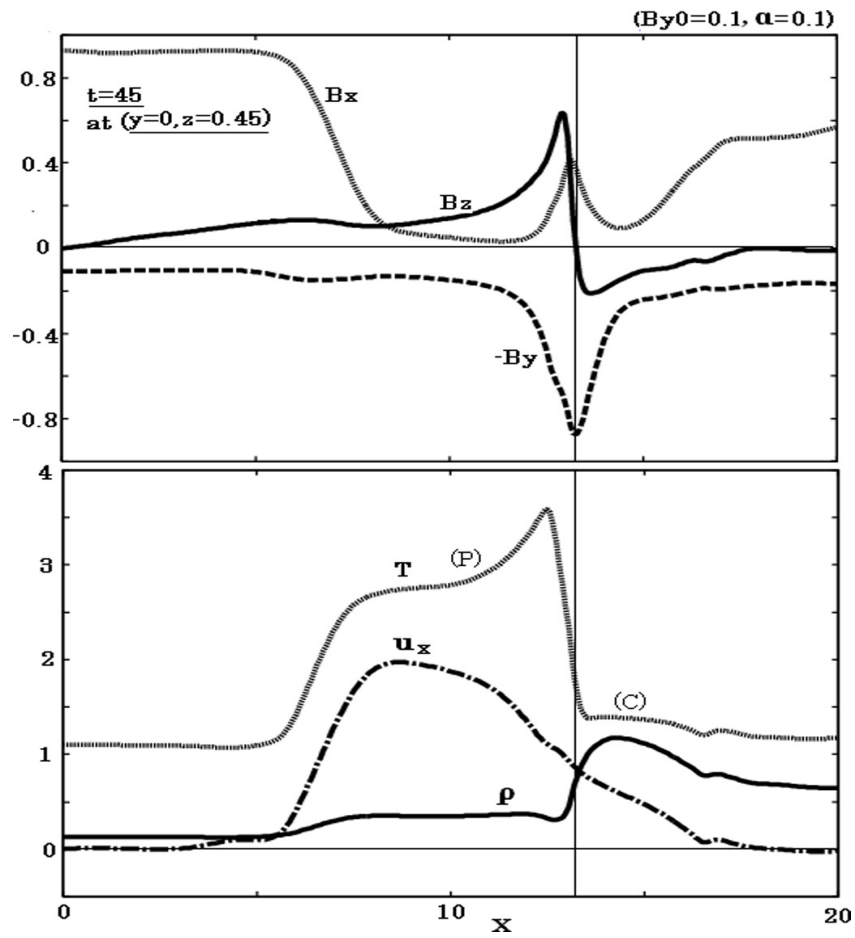

Fig. 6. Profiles of magnetic field and plasma quantities along the $\mathrm{x}$-directional line, $(x, y=0, z=0.45)$, at time $t=45$ for the case of $B_{\mathrm{y} 0}=0.1$ and $\alpha=0.1$, where $(P)$ and $(C)$ indicate the corresponding plasmoid regions.

plasmoid, where $B_{\mathrm{x}}$ is reduced and $u_{\mathrm{x}}$ is distinctly enhanced, the magnetic fields $B_{\mathrm{y}}$ and $B_{\mathrm{z}}$ are considerably small and present no definite standard profile. In general, we find that in the extent $|y| \leq 1$ the field components $B_{\mathrm{y}}$ and $B_{\mathrm{z}}$ become so large to have clear standard profiles. Therefore, in what follows, virtual satellite locations will be taken mainly in the narrow extent $|y| \leq 1$, and the results will be shown for the typical case of $B_{\mathrm{y} 0}=0.2$ and $\alpha=0$, since the results for the other cases are, at least qualitatively, quite similar.

\subsection{Virtual satellite observations}

On the basis of the plasmoid structure (Figs. 2, 4 and 5), let us examine how a virtual satellite, located in the simulation box, observes changes in quantities as the plasmoid propagates in the positive $\mathrm{x}$-direction. Here, the satellite location is assumed to be fixed in spite of the progress of time. First of all, let us consider the trajectory I, so the satellite is located in the inner part of the plasmoid around the x-axis. Figure 9 shows the magnetic fields measured by the three virtual satellites, located at $(x=9, y=0, z=0.075),(x=13, y=0, z=$ $0.075)$ and $(x=17, y=0, z=0.075)$ in the immediate vicinity of the $\mathrm{x}$-axis for the case of $B_{\mathrm{y} 0}=0.2$ and $\alpha=0$. Since the plasmoid is generated and propagated near the origin, the satellite at $x=9$ first detects the plasmoid. Then, we notice that the plasmoid, that the satellite at $x=9$ observes, is not

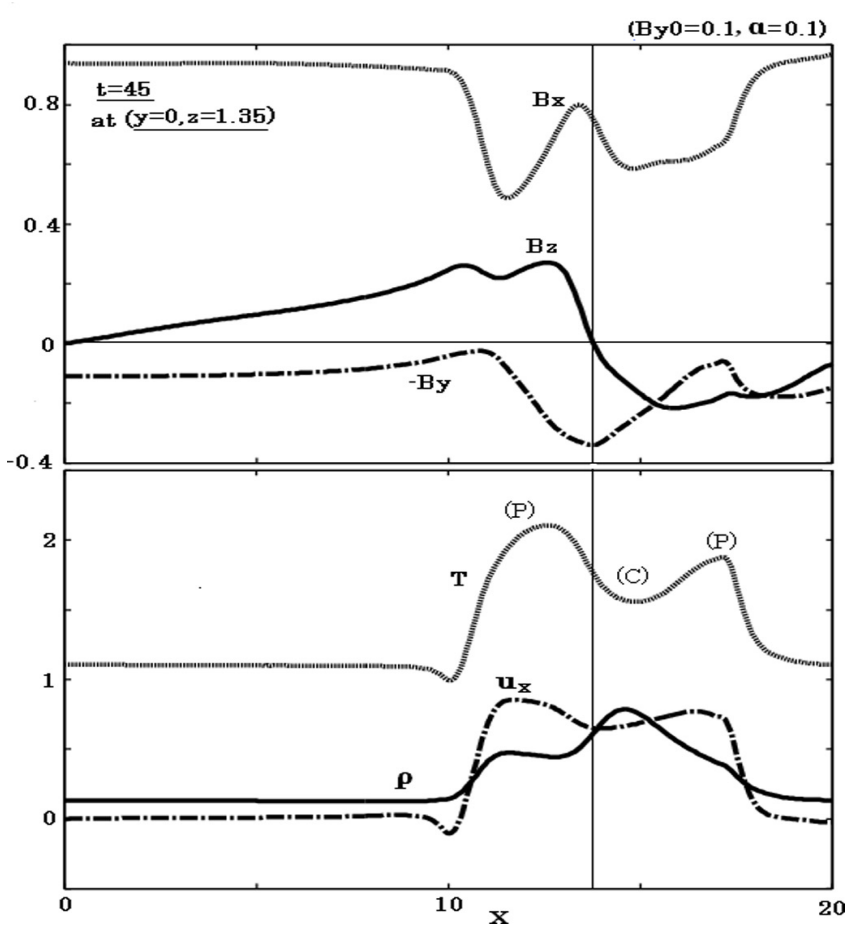

Fig. 7. Profiles of magnetic field and plasma quantities along the $\mathrm{x}$-directional line, $(x, y=0, z=1.35)$, at time $t=45$ for the case of $B_{\mathrm{y} 0}=0.1$ and $\alpha=0.1$, where $(P)$ and $(C)$ indicate the corresponding plasmoid regions.

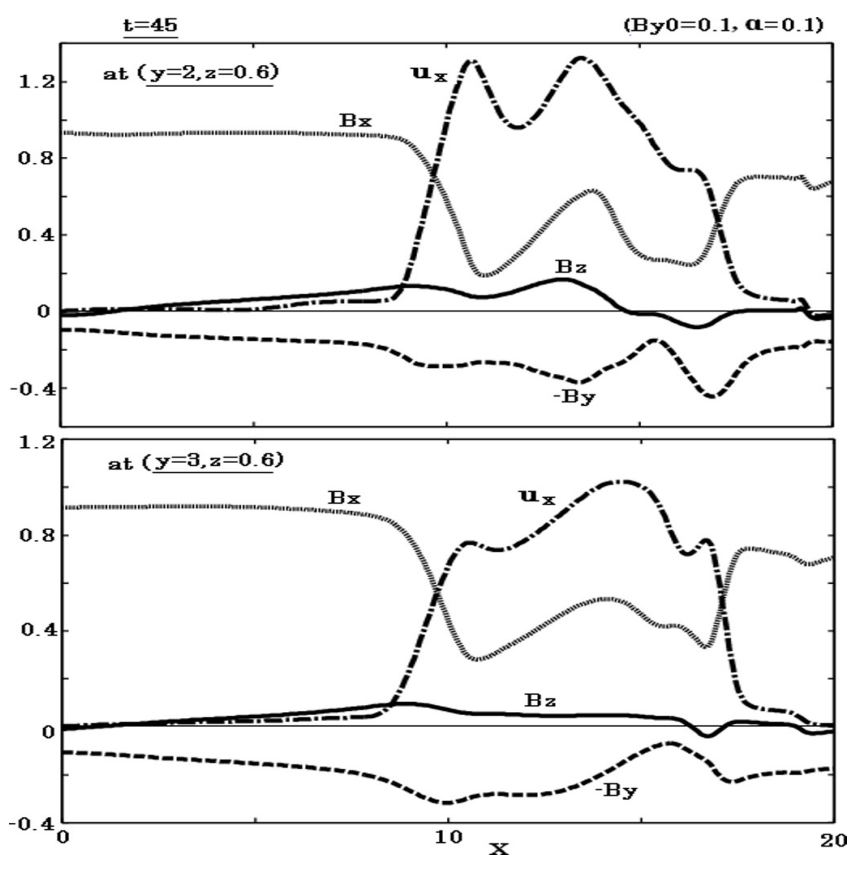

Fig. 8. Profiles of magnetic fields and flow velocity $u_{\mathrm{x}}$ along the $\mathrm{x}$ directional lines, $(x, y=2, z=0.6)$ and $(x, y=3, z=0.6)$, at time $t=45$ for the case of $B_{\mathrm{y} 0}=0.1$ and $\alpha=0.1$. 


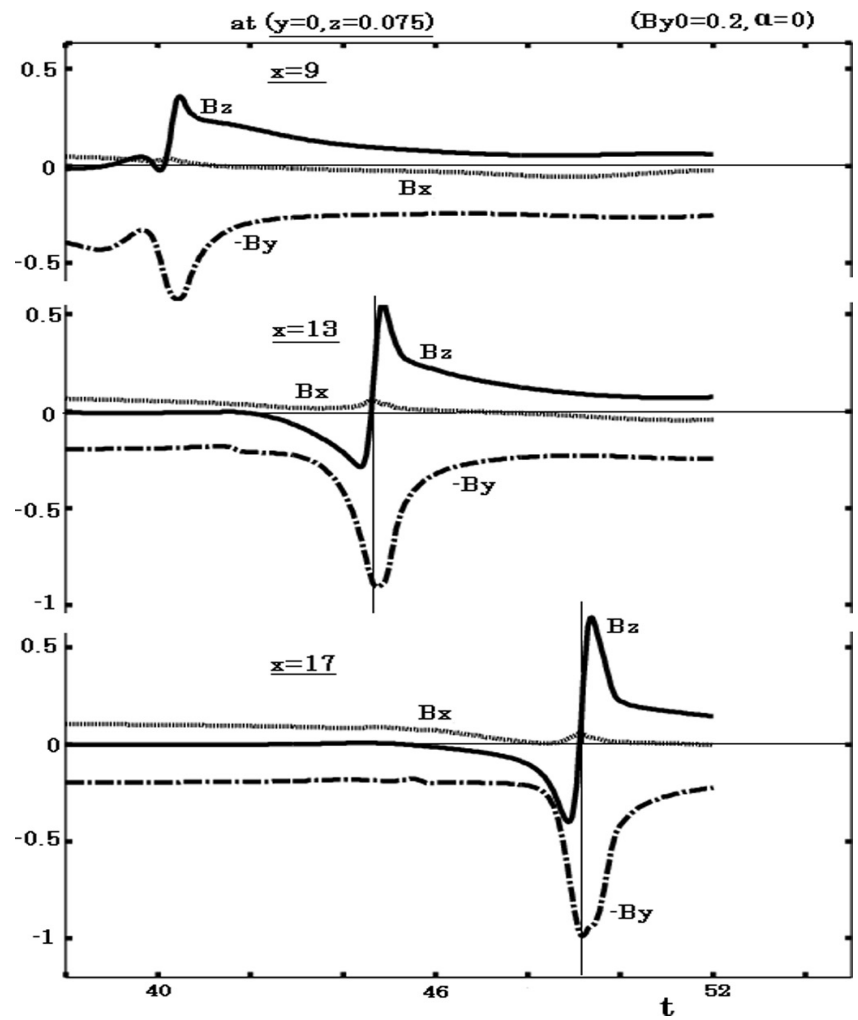

Fig. 9. Temporal variations of magnetic fields $B_{\mathrm{X}}, B_{\mathrm{y}}$ and $B_{\mathrm{Z}}$ detected by the virtual satellites located at $(x=9, y=0, z=0.075)$, $(x=13, y=0, z=0.075)$, and $(x=17, y=0, z=0.075)$ for the case of $B_{\mathrm{y} 0}=0.2$ and $\alpha=0$.

yet fully established, since the $B_{\mathrm{Z}}$ field component does not have the definite bipolar structure, but has the dipolarizationlike structure. On the other hand, the satellites located at $x=13$ and $x=17$ observe the plasmoid fully established, where the magnetic field profile is consistent with the standard one. Hence, in what follows, observations by the satellite located at $x=15$ will be shown.

Figure 10 shows the temporal changes in magnetic fields and plasma quantities observed by the virtual satellite located at $(x=15, y=0, z=0.45)$ along the trajectory I. At time $t \sim 43$, the plasmoid core region $C$ arrives at the satellite, since then the $B_{\mathrm{X}}$ field decreases and the plasma density is enhanced. As the region $\mathrm{C}$ passes away, the temperature $T$ distinctly increases and the density $\rho$ decreases, which indicates that the plasmoid reconnection region $\mathrm{P}$ arrives (Fig. 2). At the interface between the regions $P$ and $C$ the $B_{\mathrm{y}}$ field has the peak value and the $B_{\mathrm{z}}$ field changes its sign to have the definite bipolar structure. Also, Figs. 11 and 12 show the same quantities observed at the satellites located at $(x=15, y=1, z=0.45)$ and $(x=15, y=-1, z=0.45)$, respectively. In each case, the magnetic field profile is consistent with the standard one, but thermodynamic quantities are considerably different after the region $\mathrm{C}$ passes away.

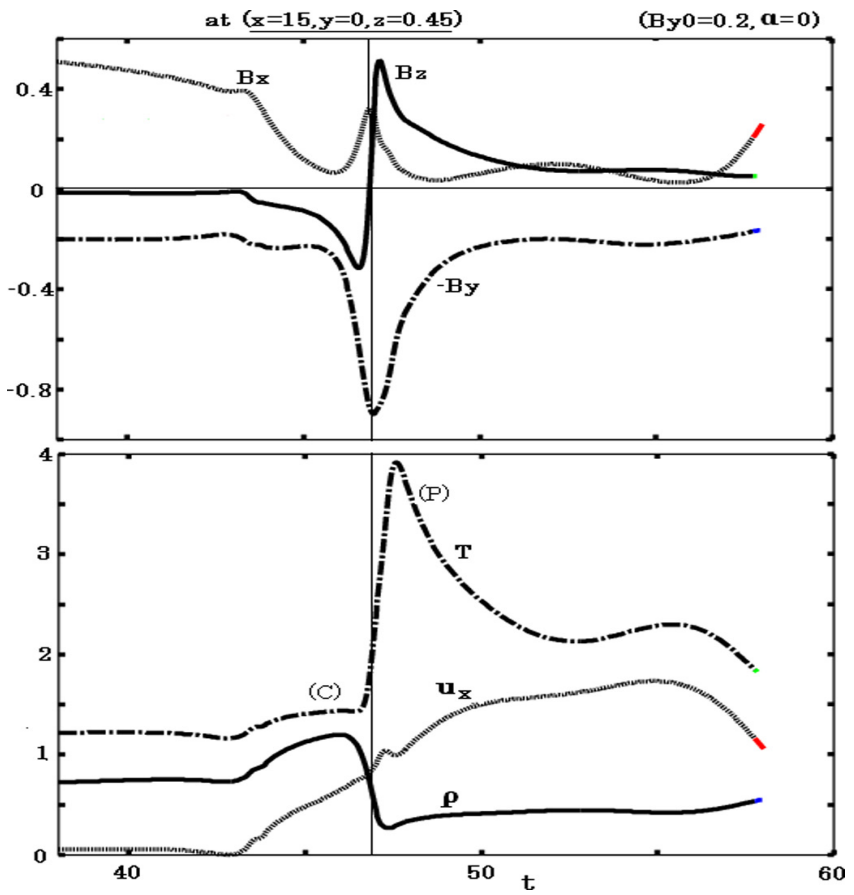

Fig. 10. Temporal variations of magnetic fields, $B_{\mathrm{X}}, B_{\mathrm{y}}$ and $B_{\mathrm{Z}}$, temperature $T$, plasma density $\rho$ and flow velocity $u_{\mathrm{x}}$ detected by the virtual satellite located at $(x=15, y=0, z=0.45)$, for the case of $B_{\mathrm{y} 0}=0.2$ and $\alpha=0$, where $(P)$ and $(C)$ indicate the corresponding plasmoid regions.

For the case of the satellite located at $y=1$, the temperature $T$ remains large with the large flow velocity $u_{\mathrm{x}}$, whereas for the cases of $y=0$ and $y=-1$ the temperature becomes distinctly reduced with the considerably smaller velocity $u_{\mathrm{x}}$. This is because region $\mathrm{P}$ shifts in the positive $\mathrm{y}$-direction, so only the satellite at $y=1$ observes the major part of the region $\mathrm{P}$, whereas the satellites at $y=0$ and -1 may observe the edge of P (Fig. 5).

Next, let us consider observations along the trajectory II (Fig. 2), so that the satellites are located in the upper part of the plasmoid (larger $z$ ). Figure 13 shows the temporal changes in magnetic fields and plasma quantities observed at $(x=15, y=0, z=1.35)$. The satellite detects the plasmoid arrival at $t \sim 42$, when both the temperature $T$ and the flow velocity $u_{\mathrm{x}}$ begin to increase notably, so the plasmoid region $\mathrm{P}$ arrives. Then, at $t \sim 44$ the temperature reduces and the plasma density increases, so the region $\mathrm{C}$ arrives. Finally, at $t \sim 47$ the temperature increases and the density decreases, so region $\mathrm{P}$ again arrives and is passing through. Hence, in this case, the plasmoid regions $\mathrm{P}, \mathrm{C}$ and $\mathrm{P}$ pass through the satellite as can be expected from Fig. 7. Compared to the case of the trajectory I (Fig. 10), the changes in quantities are more complicated, and both the temperature $T$ and the flow velocity $u_{\mathrm{x}}$ are considerably smaller, since these quantities become reduced in the upper part of the plasmoid (Fig. 4); in particular, $u_{\mathrm{x}}$ may become negative because of 


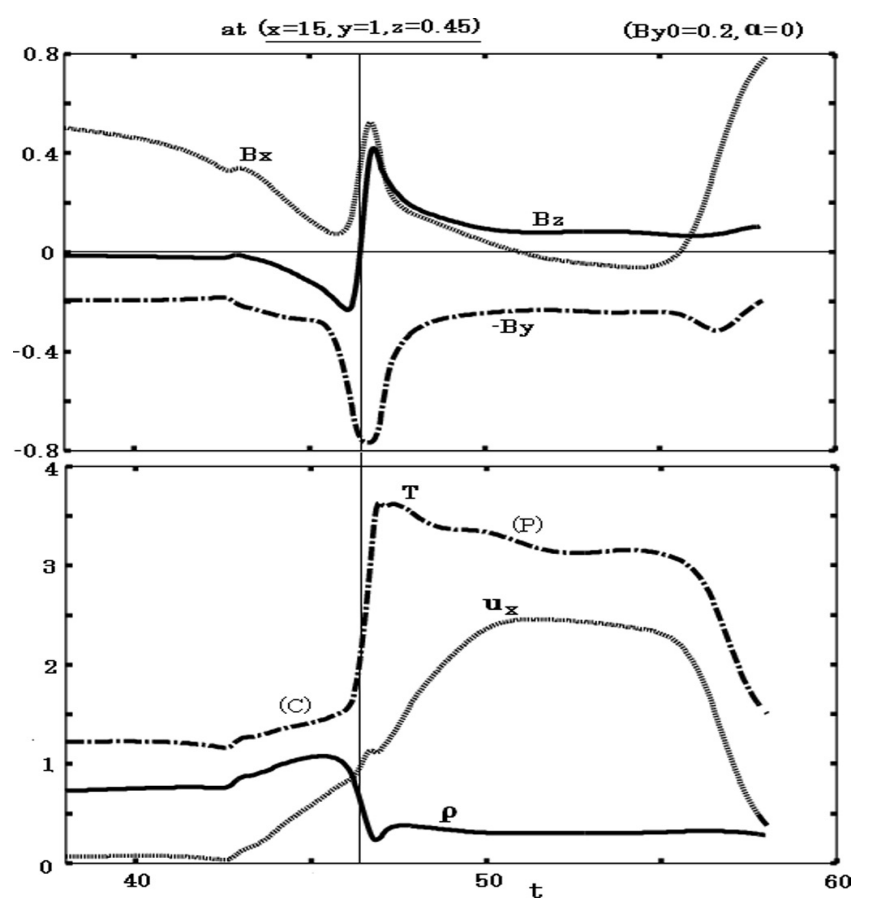

Fig. 11. Temporal variations of magnetic fields, $B_{\mathrm{x}}, B_{\mathrm{y}}$ and $B_{\mathrm{Z}}$, temperature $T$, plasma density $\rho$ and flow velocity $u_{\mathrm{x}}$ detected by the virtual satellite located at $(x=15, y=1, z=0.45)$ for the case of $B_{\mathrm{y} 0}=0.2$ and $\alpha=0$, where (P) and (C) indicate the corresponding plasmoid regions.

vortex flows around the plasmoid boundaries. However, the magnetic field profile is still consistent with the standard one, since the $B_{\mathrm{Z}}$ field changes its sign at the interface between regions $\mathrm{C}$ and $\mathrm{P}$, where the $B_{\mathrm{y}}$ field has the peak value.

In order to see the plasmoid distortion in the y-direction, Fig. 14 shows the temporal changes in the quantities observed at $(x=15, y=-1, z=1.35)$. In this case, the satellite may observe mainly the upper part of the plasmoid region $\mathrm{C}$ (Fig. 5), so that the temperature $T$ or the flow velocity $u_{\mathrm{x}}$ is not effectively enhanced. The magnetic field profile is generally consistent with the standard one, although the $B_{\mathrm{y}}$ field does not have such a sharp peak value. Similarly, Figs. 15 and 16 show the observations at $(x=15, y=1, z=1.35)$ and $(x=15, y=2, z=1.35)$, where the satellites measure mainly the plasmoid region $\mathrm{P}$ (Fig. 5), where both the temperature $T$ and the flow velocity $u_{\mathrm{x}}$ are notably enhanced. For the case of $y=1$, unlike the standard one, the time when $B_{\mathrm{y}}$ field has the peak value somewhat deviates from the time when $B_{\mathrm{Z}}$ changes its sign. For the case of $y=2, T$ and $u_{\mathrm{x}}$ become larger than those for the cases of $y=-1,0$ and 1 , which indicates that the satellite passes through the major part of the plasmoid region $\mathrm{P}$, since region $\mathrm{P}$ has shifted in the positive $y$-direction. In this case, the magnetic field profile becomes quite different from the standard one. Hence, we may recognize that when a satellite observes only the plasmoid region $\mathrm{P}$, the standard magnetic field profile (Fig. 1)

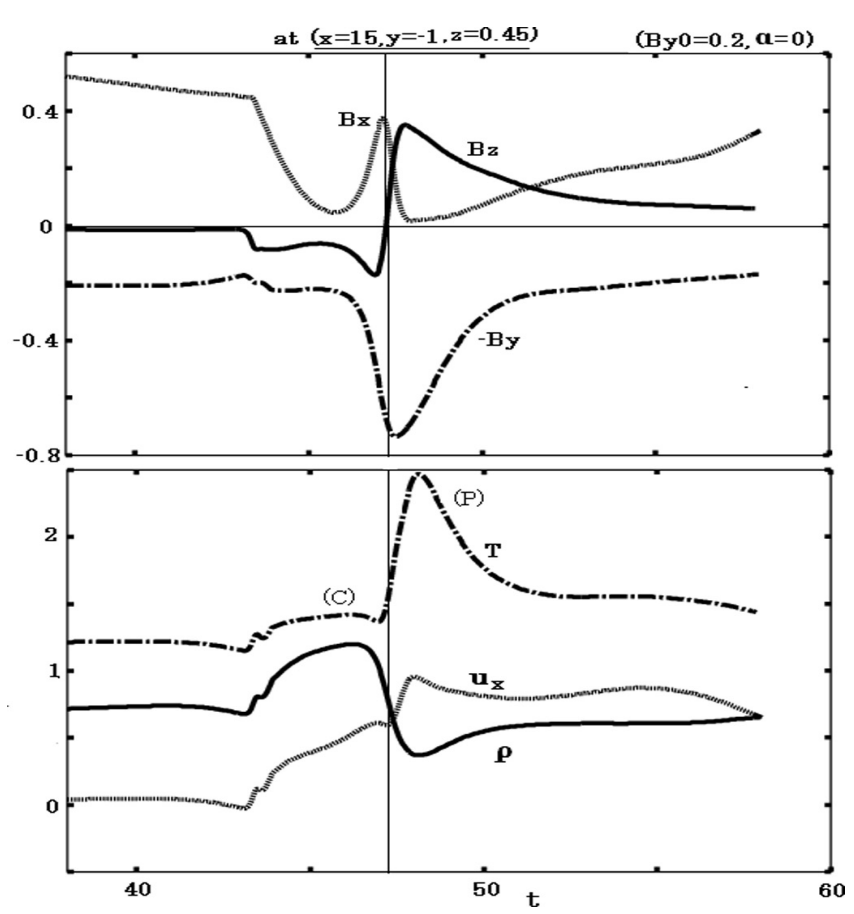

Fig. 12. Temporal variations of magnetic fields, $B_{\mathrm{x}}, B_{\mathrm{y}}$ and $B_{\mathrm{Z}}$, temperature $T$, plasma density $\rho$ and flow velocity $u_{\mathrm{X}}$ detected by the virtual satellite located at $(x=15, y=-1, z=0.45)$ for the case of $B_{\mathrm{y} 0}=0.2$ and $\alpha=0$, where (P) and (C) indicate the corresponding plasmoid regions.

becomes obscure despite that it detects apparent plamsoid signatures like enhancement of the temperature and the flow velocity $u_{\mathrm{x}}$.

\section{Summary and discussion}

On the basis of the plasmoid structure generated by the fast reconnection mechanism (Ugai, 2011), the present paper examines its propagation by virtual satellites in the simulation box. The plasmoid domain is divided into the plasmoid reconnection region $\mathrm{P}$, where magnetized plasmas with reconnected field lines, heated by reconnection dissipation mechanisms, are accumulated, and the plasmoid core region $\mathrm{C}$, where magnetized plasmas with sheared field lines, initially embedded in the current sheet and adiabatically compressed by the reconnection jet, are accumulated (Fig. 2). The observation trajectories, along which quantities are measured by the satellite, are typically divided into the trajectories I and II (Fig. 2a). In the trajectory I, the satellite is located in the inner part of the plasmoid around the plasmoid central axis (x-axis). In this case, the region $\mathrm{C}$ first arrives at the satellite position, and subsequently region $\mathrm{P}$ passes through. The observed magnetic fields (Figs. 9-12) are in good agreement with the standard magnetic field profile detected by the actual spacecraft (Fig. 1). However, if the satellite $x$ position is too 


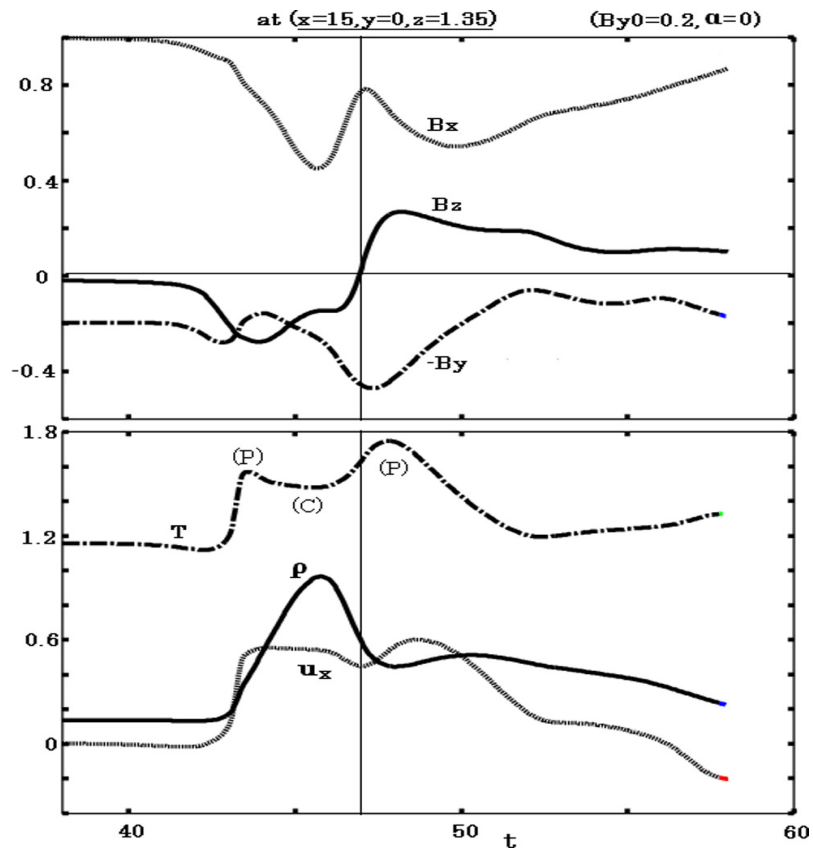

Fig. 13. Temporal variations of magnetic fields, $B_{\mathrm{X}}, B_{\mathrm{y}}$ and $B_{\mathrm{Z}}$, temperature $T$, plasma density $\rho$ and flow velocity $u_{\mathrm{x}}$ detected by the virtual satellite located at $(x=15, y=0, z=1.35)$ for the case of $B_{\mathrm{y} 0}=0.2$ and $\alpha=0$, where (P) and (C) indicate the corresponding plasmoid regions.

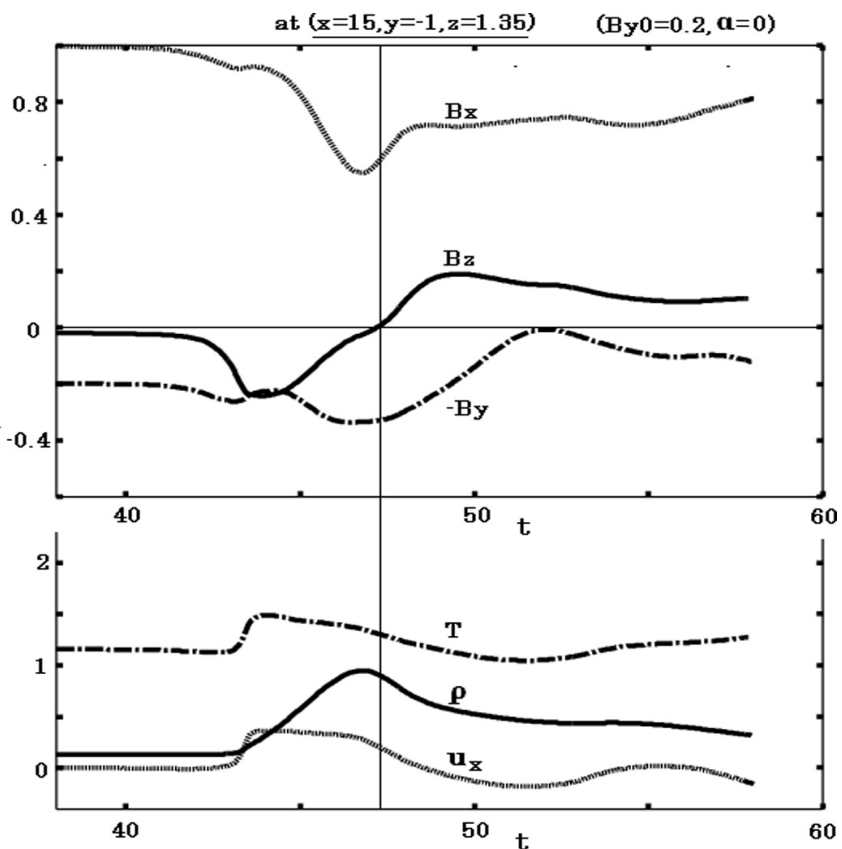

Fig. 14. Temporal variations of magnetic fields, $B_{\mathrm{X}}, B_{\mathrm{y}}$ and $B_{\mathrm{Z}}$, temperature $T$, plasma density $\rho$ and flow velocity $u_{\mathrm{X}}$ detected by the virtual satellite located at $(x=15, y=-1, z=1.35)$ for the case of $B_{\mathrm{y} 0}=0.2$ and $\alpha=0$.

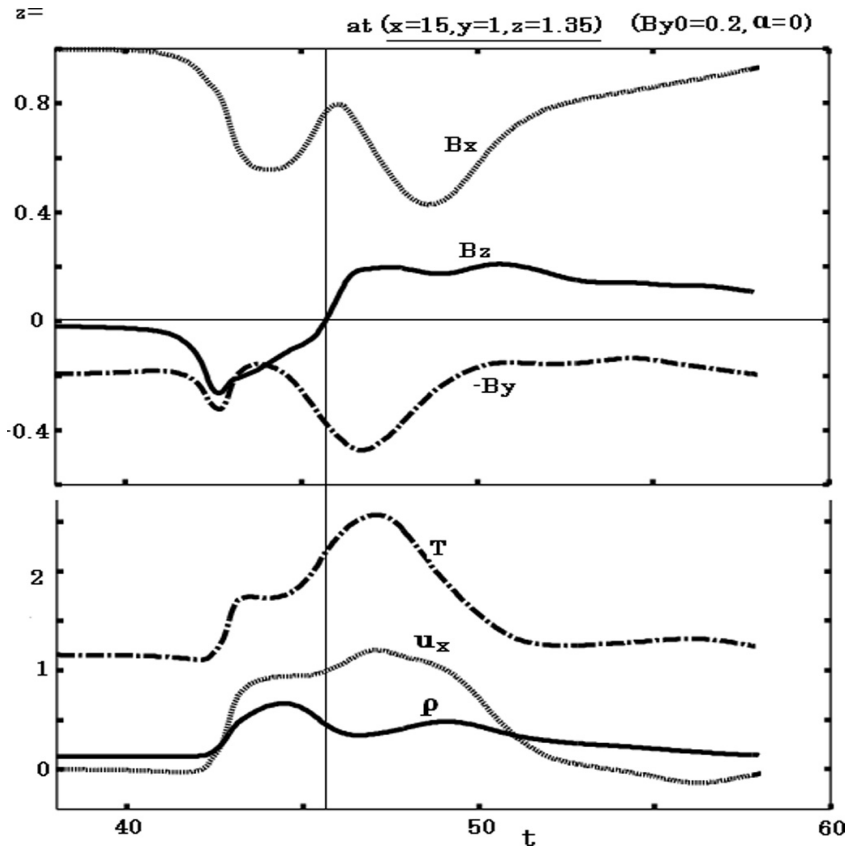

Fig. 15. Temporal variations of magnetic fields, $B_{\mathrm{X}}, B_{\mathrm{y}}$ and $B_{\mathrm{Z}}$, temperature $T$, plasma density $\rho$ and flow velocity $u_{\mathrm{x}}$ detected by the virtual satellite located at $(x=15, y=1, z=1.35)$ for the case of $B_{\mathrm{y} 0}=0.2$ and $\alpha=0$.

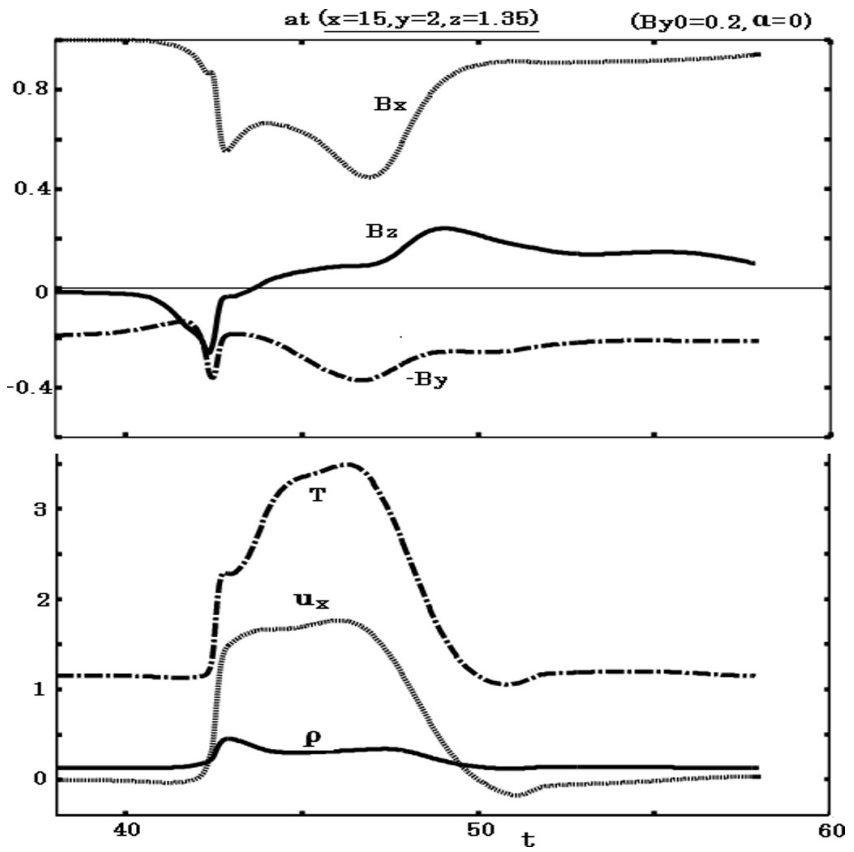

Fig. 16. Temporal variations of magnetic fields, $B_{\mathrm{X}}, B_{\mathrm{y}}$ and $B_{\mathrm{Z}}$, temperature $T$, plasma density $\rho$ and flow velocity $u_{\mathrm{x}}$ detected by the virtual satellite located at $(x=15, y=2, z=1.35)$ for the case of $B_{\mathrm{y} 0}=0.2$ and $\alpha=0$. 


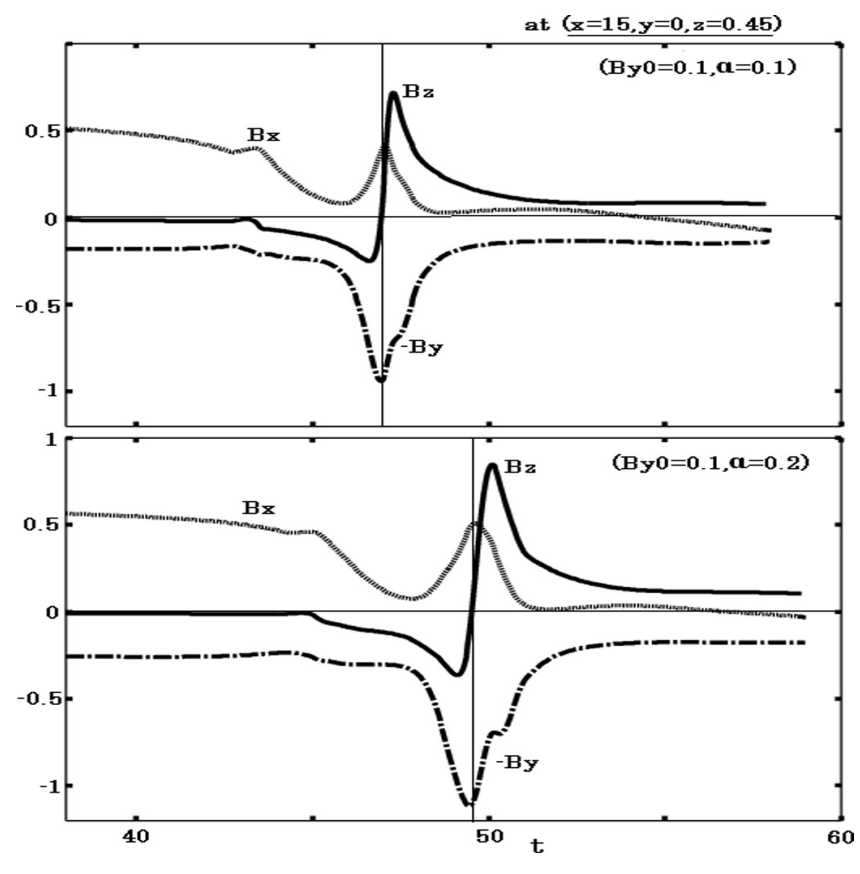

Fig. 17. Temporal variations of magnetic fields, $B_{\mathrm{X}}, B_{\mathrm{y}}$ and $B_{\mathrm{Z}}$ measured by the virtual satellite located at $(x=15, y=0, z=0.45)$, for the other cases of $\left(B_{\mathrm{y} 0}=0.1, \alpha=0.1\right)$ and $\left(B_{\mathrm{y} 0}=0.1, \alpha=0.2\right)$.

close to the $X$ neutral line, where magnetic reconnection occurs, it observes the plasmoid that is not fully established; in particular, the $B_{\mathrm{z}}$ field does not yet have the definite bipolar structure (Fig. 9).

Regarding the trajectory II, the satellite is located in the upper part of the plasmoid, and it may subsequently observe the plasmoid regions $\mathrm{P}, \mathrm{C}$ and $\mathrm{P}$. The observed magnetic fields are generally consistent with the standard field profile (Fig. 13), although changes in quantities become more complicated, and the temperature and the flow velocity are considerably smaller than those for the trajectory I. Since the plasmoid region $\mathrm{P}$ is shifted in the positive y-direction in $z>0$, the satellites located at the larger $y$ positions observe only region $\mathrm{P}$ (Fig. 5). The observed magnetic fields no longer have the definite standard profile despite that the temperature and the flow velocity are significantly enhanced. In general, in the y-directional narrow channel, say in $|y|<1$, the observed magnetic fields are in good agreement with the standard plasmoid field profile (Fig. 1), since the fast reconnection jet region is significantly limited in the narrow channel in the y-direction (Fig. 4).

Unlike the traditional concept that the magnetic field lines inside the plasmoid should be helical (or closed), we demonstrate that the standard magnetic field profile (Fig. 1) does not necessarily mean helical field lines inside the plasmoid; in fact, inside the plasmoid (Fig. 2) the reconnected field lines in $x<X_{\mathrm{C}}$ cannot be topologically connected to the sheared field lines in $x>X_{\mathrm{C}}$. In order to see this more clearly, let us consider the typical field profile shown in Figs. 9 and 10; also, in view of generality, Fig. 17 shows the temporal variations of magnetic fields detected by the satellite located at $(x=15, y=0, z=0.45)$ for the other two cases, $\left(B_{\mathrm{y} 0}=0.1\right.$, $\alpha=0.1)$ and $\left(B_{\mathrm{y} 0}=0.1, \alpha=0.2\right)$. These field profiles seem almost completely consistent with those detected by actual spacecrafts, such as those shown in Figs. $2 \mathrm{~b}$ and 3 in Slavin et al. (2003) and in Fig. 2 in Sormakov and Sergeev (2008). In these cases, the bipolar structure of the $B_{\mathrm{Z}}$ field looks more symmetric, so this magnetic field profile is considered to be an apparent evidence of the force-free flux rope with helical field lines due to multiple reconnections (Slavin et al., 2003), whereas Sormakov and Sergeev (2008) pointed out that the field lines should not be helical inside the plasmoid. The present virtual satellite observations demonstrate that the flux-rope-like field profile is the direct outcome of the plasmoid generated by the single fast reconnection evolution (Fig. 2).

More quantitatively, the normalized quantities may be applied to actual geomagnetic tail situations. Here, let us take the unit velocity $V_{\mathrm{Ax} 0}=B_{\mathrm{x} 0} / \sqrt{\mu_{0} \rho_{i}} \sim 500 \mathrm{~km} \mathrm{~s}^{-1}$, where $\rho_{i}$ is the plasma density measured at the initial plasma sheet centre $(z=0)$, and the half thickness of the initial plasma sheet $d_{0} \sim 5000 \mathrm{~km}$ immediately before the reconnection onset. Then, the unit time is $d_{0} / V_{\mathrm{Ax} 0} \sim 10 \mathrm{~s}$ for the present simulation model. We may then see that virtual satellite observations are generally consistent with actual satellite observations (e.g., Fig. 1) both qualitatively and quantitatively. In the tail lobe region outside the plasmoid too, we recently demonstrated that satellite observations of the so-called travelling compression regions (TCRs) (Slavin et al., 1993) can be exactly explained both qualitatively and quantitatively by the plasmoid generated by the fast reconnection mechanism (Ugai and Zheng, 2006; Ugai, 2010). Hence, we may conclude that the spontaneous fast reconnection model is responsible for the plasmoids and TCRs observed in the geomagnetic tail.

Finally, let us consider some basic assumptions adopted in the present study. When a magnetic neutral sheet with $B_{\mathrm{Z}} \sim 0$ is formed somewhere in the tail and becomes sufficiently thin, magnetic reconnection drastically builds up and proceeds symmetrically. Then, if the northward field component $B_{\mathrm{Z}}>0$, neglected here, is assumed to exist outside the neutral sheet, the sheared field lines with $B_{\mathrm{Z}}>0$, initially embedded in the current sheet, will be accumulated in the plasmoid core region C. Hence, the initial $B_{\mathrm{Z}}>0$ should be added to the field components in the region $\mathrm{C}$, where $B_{\mathrm{Z}}<0$ for the present earthward moving plasmoid (Fig. 10). For the tailward moving plasmoid (for $x<0$ ), $B_{\mathrm{Z}}>0$ in region C since $B_{\mathrm{Z}}(-x, y=z=0)=-B_{\mathrm{Z}}(x, y=z=0)$, so the initial northward field may tend to enhance $B_{\mathrm{Z}}>0$ in $\mathrm{C}$ as seen in actual satellite observations (Ieda et al., 1998), although the plasmoid will significantly change its structure (in particular, the thermodynamic quantities) as it propagates in the distant tail. Also, the temperature $T$ is assumed to 
be initially constant everywhere unlike the actual geomagnetic tail. In this respect, we already studied the plasmoid dynamics for different initial temperature distributions to examine the TCRs and found that the basic 3-D magnetic field structure is not influenced (Ugai and Zheng, 2006). In addition, we assume the MHD model, which neglects particle effects like those of finite Larmor radius; in fact, these effects may affect the detailed fine structure of the diffusion region and the plasmoid region. Also, besides the present principal plasmoid, small-scale magnetic islands are generated in the active $\mathrm{X}$ reconnection (diffusion) region and may propagate in the tailward or earthward direction (Ugai, 1985; Ugai and Zheng, 2006), which is not considered in the present study. Hence, in understanding more details of complicated substorm observations, these points should also be taken into account relevantly.

Acknowledgements. This work was supported by the Grant-in-Aids (21340142) from the Ministry of Education in Japan, Mitsubishi Foundation, RISH of Kyoto University, and Solar-Terrestrial Environment Laboratory of Nagoya University.

Topical Editor R. Nakamura thanks two anonymous referees for their help in evaluating this paper.

\section{References}

Hones Jr., E. W.: Substorm processes in the magnetotail: Comments on "On hot tenuous plasma, fireballs, and boundary layers in the Earth's magnetotail” by L. A. Frank et al., J. Geophys. Res., 82, 5633-5640, 1977.

Ieda, A., Machida, S., Mukai, T., Saito, Y., Yamamoto, T., Nishida, A., Terasawa, T., and Kokubun, S.: Statistical analysis of the plasmoid evolution with Geotail observations, J. Geophys. Res., 103, 4453-4465, 1998.

Ji, H., Yamada, M., Hsu, S., and Kulsrud, R.: Experimental test of the Sweet-Parker model of magnetic reconnection, Phys. Rev. Lett., 80, 3256-3259, 1998.

Lui, A. T. Y.: A multiscale model for substorms, Space Sci. Rev., 95, 325-345, 2001.

Lui, A. T. Y.: Potential plasma instabilities for substorm expansion onsets, Space Sci. Rev., 113, 127-206, 2004.

Nakamura, R., Baumjohann, W., Asano, Y., Runov, A., Balogh, A., Owen, C. J., Fazakerley, A. N., Fujimoto, M., Klecker, B., and Reme, H.: Dynamics of thin current sheets associated with magnetotail reconnection, J. Geophys. Res., 111, A11206, doi:10.1029/2006JA011706, 2006.

Nitta, S.: Outflow structure and reconnection rate of the self-similar evolution model of fast magnetic reconnection, Astrophys. J., 610, 1117-1128, 2004.

Ono, Y., Inomoto, M., Ueda, Y., Matsuyama, T., and Murata, Y.: Fast compression of a current sheet during externally driven magnetic reconnection, Earth Planets and Space, 53, 521-526, 2001.

Petkaki, P. and Freeman, M. P.: Nonlinear dependence of anomalous ion-acoustic resistivity on electron drift velocity, Astrophys. J., 686, 686-693, 2008.

Petschek, H. E.: Magnetic field annihilation, in AAS-NASA Symposium on the Physics of Solar Flares, NASA Spec. Pub., SP-50, 425-439, 1964.
Priest, E. R. and Forbes, T. G.: New models for fast steady state magnetic reconnection, J. Geophys. Res., 91, 5579-5588, 1986.

Priest, E. R. and Forbes, T. G.: Magnetic reconnection: MHD theory and applications, Cambridge University Press, Cambridge, UK, 2000.

Runov, A., Nakamura, R., Baumjohann, W., Treumann, R. A., Zhang, T. L., Volwerk, M., Voros, Z., Balogh, A., Glasmeier, K.H., Klecker, B., Reme, H., and Kistler, L.: Current sheet structure near magnetic X-line observed by Cluster, Geophys. Res. Lett., 30, 1579, doi:10.1029/2002GL0160730, 2003.

Sergeev, V. A., Elphic, R. C., Mozer, F. S., Saint-Marc, A., and Sauvaud, J. A.: A two-satellite study of nightside flux transfer events in the plasma sheet, Planet. Space Sci., 40, 1551-1572, 1992.

Slavin, J. A., Smith, M. F., Mazur, E. L., Baker, D. N., Hones Jr., E. W., Iyemori, T., and Greenstadt, E. W.: ISEE 3 observations of traveling compression regions in the Earth's magnetotail, J. Geophys. Res., 98, 15425-15446, 1993.

Slavin, J. A., Lepping, R. P., Gjerloev, J., Fairfield, D. H., Hesse, M., Owen, C. J., Moldwin, M. B., Nagai, T., Ieda, A., and Mukai, T.: Geotail observations of magnetic flux ropes in the plasma sheet, J. Geophys. Res., 108, 1015, doi:10.1029/2002JA009557, 2003.

Sormakov, D. A. and Sergeev, V.: Topology of magnetic flux ropes in the magnetospheric plasma sheet as measured by the Geotail satellite, Cosmic Res., 46, 387-391, 2008.

Ugai, M.: Temporal evolution and propagation of a plasmoid associated with asymmetric fast magnetic reconnection, J. Geophys. Res., 90, 9576-9582, 1985.

Ugai, M.: Global dynamics and rapid collapse of an isolated current-sheet system enclosed by free boundaries, Phys. Fluids, 29, 3659-3667, 1986.

Ugai, M.: Computer studies of a large-scale plasmoid driven by spontaneous fast reconnection, Phys. Fluids B, 1, 942-948, 1989.

Ugai, M.: Computer studies on development of the fast reconnection mechanism for different resistivity models, Phys. Fluids B, 4, 2953-2963, 1992.

Ugai, M.: Computer studies on plasmoid dynamics associated with the spontaneous fast reconnection mechanism, Phys. Plasmas, 2, 3320-3328, 1995.

Ugai, M.: Computer studies on the spontaneous fast reconnection model as a nonlinear instability, Phys. Plasmas, 6, 1522-1531, 1999.

Ugai, M.: The evolution of fast reconnection in a threedimensional current sheet system, Phys. Plasmas, 15, 082306, doi:10.1063/1.2969737, 2008.

Ugai, M.: Magnetic field structure of large-scale plasmoid generated by the fast reconnection mechanism in a sheared current sheet, Ann. Geophys., 28, 1511-1521, doi:10.5194/angeo-281511-2010, 2010.

Ugai, M.: The structure and dynamics of a large-scale plasmoid generated by fast reconnection in the geomagnetic tail, Ann. Geophys., 29, 147-156, doi:10.5194/angeo-29-147-2011, 2011.

Ugai, M. and Tsuda, T.: Magnetic field-line reconnexion by localized enhancement of resistivity. Part 1 . Evolution in a compressible MHD fluid, J. Plasma Phys., 17, 337-356, 1977.

Ugai, M. and Zheng, L.: Parametric studies on traveling compression regions observed in the Earth's magnetotail, Phys. Plasmas, 13, 062906, doi:10.1063/1.2212827, 2006. 
Vasyliunas, V. M.: Theoretical models of magnetic field line merging, Rev. Geophys. Space Phys., 13, 303-336, 1975.
Zenitani, S. and Miyoshi, T.: Magnetohydrodynamic structure of a plasmoid in fast reconnection in low-beta plasmas, Phys. Plasmas, 18, 022105, doi:10.1063/1.3554655, 2011. 